\title{
Congenital Self-Healing Langerhans Cell Histiocytosis: A Rare Presentation of Blueberry Muffin Baby "Spectrum"
}

\author{
Katharina Hansel ${ }^{a} \quad$ Marta Tramontana $^{a} \quad$ Stefania Troiani $^{b} \quad$ Diletta de Benedictis $^{b}$ \\ Leonardo Bianchi $^{a}$ Rosa Cucchia ${ }^{a}$ Stefano Simonettia ${ }^{a}$ Luca Stingeni ${ }^{a}$ \\ a Section of Dermatology, Department of Medicine, University of Perugia, Perugia, Italy; \\ ${ }^{b}$ Division of Neonatology and Neonatal Intensive Care Unit, Department of Maternal and \\ Child Health, Santa Maria della Misericordia Hospital of Perugia, Perugia, Italy
}

\section{Keywords}

Congenital self-healing Langerhans cell histiocytosis · Hashimoto-Pritzker disease ·

Blueberry muffin baby

\begin{abstract}
A case of congenital self-healing Langerhans cell histiocytosis (CSHLCH), also known as Hashimoto-Pritzker disease, is reported. The newborn presented as blueberry muffin baby at birth, showing numerous non-blanching blue-purplish and dark-red papular, nodular lesions without documented infections and systemic involvement. Histopathological and immunohistochemical findings were suggestive for Langerhans cell histiocytosis. During the first 12 weeks of life, the cutaneous lesions progressively and spontaneously regressed with some atrophic scars. One-year follow-up is negative for relapse of cutaneous lesions or systemic involvement, confirming the diagnosis of CSHLCH.

\section{Introduction}

Congenital self-healing Langerhans cell histiocytosis (CSHLCH), also known as Hashimoto-Pritzker disease [1], is a rare benign type of Langerhans cell histiocytosis (LCH) [2]. It occurs at birth or during the neonatal period and is characterized by red-brown macular, papular, or nodular lesions with a tendency to spontaneous regression within a few months. Skeletal, lung, ocular, and abdominal involvements are rare [3]. We report an unusual case of CSHLCH presenting as blueberry muffin baby (BMB) and self-healing within 3 months. 

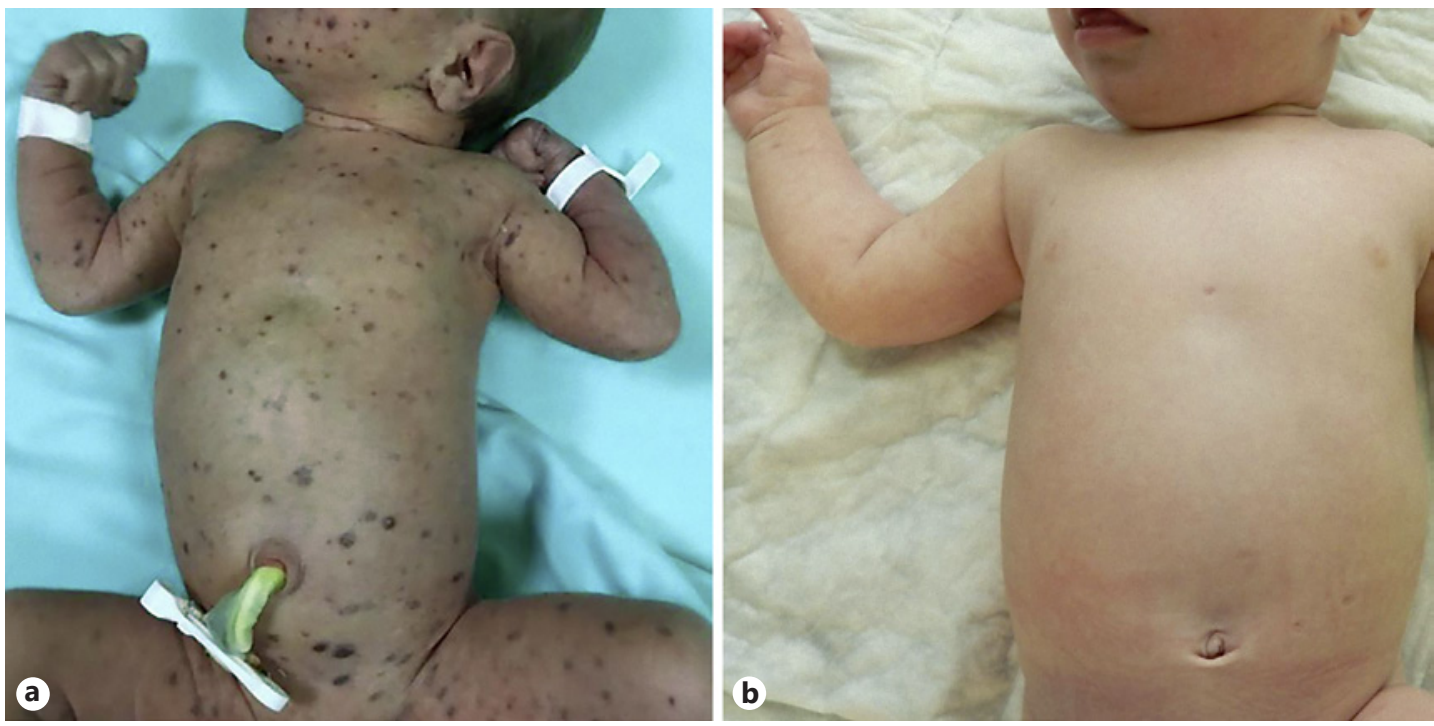

Fig. 1. a Blue-purplish and dark-red papular and nodular lesions, involving the face, scalp, trunk, and limbs. b The infant at the age of 3 months: all skin lesions were completely regressed, some by leaving atrophic scars.

\section{Case History}

A full-term male neonate born spontaneously after an uncomplicated pregnancy showed numerous 1- to 5-mm, non-blanching blue-purplish and dark-red papular and nodular lesions at birth (Fig. 1a). These firm and non-tender lesions were disseminated over the face, scalp, trunk, and limbs, sparing mucous membranes, as observed in BMB. The baby was otherwise healthy, and laboratory tests with complete blood cell count, routine serum biochemical analyses including kidney and liver function tests, clotting tests, and C-reactive protein were within a normal range. Serology for TORCH (toxoplasmosis, rubella, cytomegalovirus, and herpes) group infections, Epstein-Barr virus, coxsackie virus A and B, adenovirus, parvovirus, and syphilis were negative. Peripheral blood smear revealed normal white blood cell and platelet count with lymphocyte activation but no evidence of immature cells. Ophthalmologic and audiology screenings were normal.

A skin biopsy on a reddish-blue papulonodular lesion on the left thigh was performed. Histopathology showed the presence of a dermal infiltrate of histiocytes with occasional multinuclear giant cells (Fig. 2a); immunohistochemistry showed positivity for CD1a (Fig. 2b), CD207/Langerin (Fig. 2c), S-100, and CD68/KP1. Histopathological and immunohistochemical findings, and particularly positivity for CD207/Langerin and CD1a, were suggestive for LCH [2]. Therefore, radiographic skeletal surveys and lung ultrasound scans were performed, resulting negative. Abdominal ultrasound revealed only multiple reactive lymph nodes. The absence of systemic involvement allowed us to make a presumptive diagnosis of CSHLCH, and a "wait-and-see" approach with a long-term weekly follow-up was chosen. During the first 12 weeks of life, a progressive and spontaneous regression of the cutaneous lesions was observed, and only some atrophic scars were present (Fig. 1b).

To date, the child has been followed clinically for 12 months without any evidence of relapse of cutaneous lesions or systemic involvement, confirming the diagnosis of CSHLCH. 

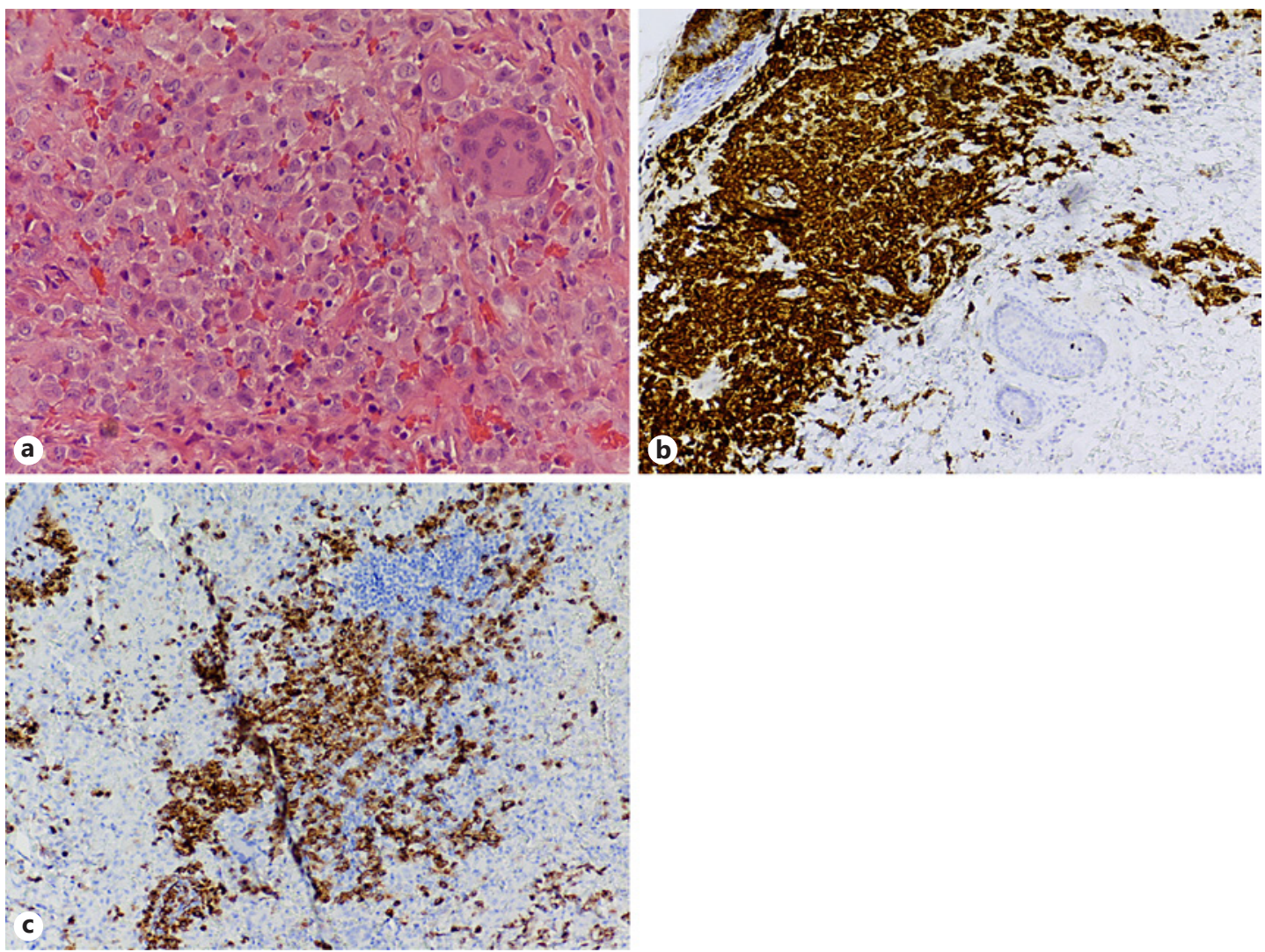

Fig. 2. a Dermal infiltrate of histiocytes with evidence of a multinuclear giant cell. H\&E. $\times 400$. Diffuse expression of CD1a (b) and CD207/Langerin (c) in the dermal infiltrate (streptavidin-biotin $\times 100$ ).

\section{Discussion}

CSHLCH is a part of the clinical spectrum of LCH. LCH is actually classified by the involvement of single or multiple organ systems, single or multiple sites within a particular organ system, and by the presence of risk-organ involvement, especially the liver, spleen, and bone marrow [4]. CSHLCH is typically a single-system LCH, as observed in our patient, presenting at birth or appearing in the neonatal period with solitary or multiple cutaneous lesions, with usually a benign and self-limiting course $[3,5]$. Rarely, CSHLCH has the clinical presentation of BMB, a term coined in the 1960s to describe cutaneous manifestations in newborns with rubella infection [6]. It is characterized by generalized eruption of nonblanching, blue-purpuric macular, papular, or nodular lesions, and represents postnatal expression of fetal extramedullary hematopoiesis in the dermis, which normally persists during embryologic development until the fifth month of gestation. Several causes have been identified, not only in association with the TORCH group intrauterine infections but also with hematological, autoimmune, and proliferative disorders, including CSHLCH [7]. According to Cano Barà et al. [8], we prefer to consider CSHLCH a rare presentation among the BMB "spectrum" and not a disease "presenting as" [9] or "mimicking" [10] BMB. Also considering our case, 11 cases of LCH presenting as BMB have been reported [8]. Nine out of these were expression of CSHLCH (including our case), while only 2 presented with systemic involvement.

In conclusion, our observation emphasizes that neonatal CSHLCH may clinically present as BMB. Exclusion of infections and systemic involvement as well as histopathological and 
immunohistochemical findings are crucial to diagnose CSHLCH and therefore to permit the "wait-and-see" approach. In fact, in our case, a close follow-up allowed us to avoid unnecessary and potentially harmful treatments, but a continued long-term follow-up is mandatory.

\section{Statement of Ethics}

The study protocol has been approved by the institute's committee on human research. Subjects have given their informed consent.

\section{Disclosure Statement}

The authors have no conflicts of interest to declare.

\section{Funding Sources}

No funding was received.

\section{References}

1 Hashimoto K, Pritzker MS. Electron microscopic study of reticulohistiocytoma. An unusual case of congenital, self-healing reticulohistiocytosis. Arch Dermatol. 1973 Feb;107(2):263-70.

2 Allen CE, Merad M, McClain KL. Langerhans-Cell Histiocytosis. N Engl J Med. 2018 Aug;379(9):856-68.

3 Hashimoto K, Bale GF, Hawkins HK, Langston C, Pritzker MS. Congenital self-healing reticulohistiocytosis (Hashimoto-Pritzker type). Int J Dermatol. 1986 Oct;25(8):516-23.

4 Krooks J, Minkov M, Weatherall AG. Langerhans cell histiocytosis in children: History, classification, pathobiology, clinical manifestations, and prognosis. J Am Acad Dermatol. 2018 Jun;78(6):1035-44.

5 Mandel VD, Ferrari C, Cesinaro AM, Pellacani G, Del Forno C. Congenital “self-healing” Langerhans cell histiocytosis (Hashimoto-Pritzker disease): a report of two cases with the same cutaneous manifestations but different clinical course. J Dermatol. 2014 Dec;41(12):1098-101.

6 Mehta V, Balachandran C, Lonikar V. Blueberry muffin baby: a pictoral differential diagnosis. Dermatol Online J. 2008 Feb;14(2):8.

7 Morren MA, Vanden Broecke K, Vangeebergen L, Sillevis-Smitt JH, Van Den Berghe P, Hauben E, et al. Diverse Cutaneous Presentations of Langerhans Cell Histiocytosis in Children: A Retrospective Cohort Study. Pediatr Blood Cancer. 2016 Mar;63(3):486-92.

8 Cano Bará S, Vargas-Navia N, Rueda Plata R. Case of congenital self-healing reticulohistiocytosis expanding the spectrum of Blueberry Muffin Baby. Am J Dermatopathol. 2018 Feb;40(2):136-8.

9 Popadic S, Brasanac D, Arsov B, Nikolic M. Congenital self-healing histiocytosis presenting as blueberry muffin baby: a case report and literature review. Indian J Dermatol Venereol Leprol. 2012 May-Jun;78(3):407.

10 Sankilampi U, Huikko-Tarvainen S, Kärjä V, Pirinen E, Naukkarinen A, Hollmén A. Congenital Langerhans cell histiocytosis mimicking a "blueberry muffin baby". J Pediatr Hematol Oncol. 2008 Mar;30(3):245-8. 